\title{
Survey Science with the SKA Molongo Pathfinder
}

\author{
A. J. Green ${ }^{* \dagger}$ \\ Sydney Institute for Astronomy, School of Physics, University of Sydney, NSW Australia \\ E-mail: anne.green@sydney .edu.au

\section{G.J. Madsen, D. Campbell-Wilson, D. Thakkar, J.Banyer, R.W. Hunstead} \\ Sydney Institute for Astronomy, School of Physics, University of Sydney, NSW Australia
}

\begin{abstract}
The Square Kilometre Array Molonglo Prototype (SKAMP) is a project using innovative technologies to transform the scientific capabilities of the University of Sydney's Molonglo Radio Telescope, located outside Canberra. SKAMP is trialling technologies and concepts as a pathfinder project for the Square Kilometre Array. The project has the goal to replace the whole signal chain with wide-band digital devices to produce a dual-polarisation 12,480-channel spectrometer operating in the band $700-1200 \mathrm{MHz}$ with an instantaneous operating bandwidth of about $95 \mathrm{MHz}$. The angular resolution of the telescope will be unchanged at about $45^{\prime \prime}$. The spectral resolution will be $7.5 \mathrm{kHz}$ per channel $\left(2.5 \mathrm{~km} \mathrm{~s}^{-1}\right.$ at $\left.843 \mathrm{MHz}\right)$ and the continuum source sensitivity will be about $200 \mu \mathrm{Jy}_{\text {beam }}{ }^{-1}$. The project is currently completing the second stage, to enable a spectral line capability. Three key science projects from this second stage (SKAMP-2) will be characterising the dynamic radio sky, a survey of red-shifted HI to test models of galaxy assembly and studies of the ionised interstellar medium. These surveys and current progress are described.
\end{abstract}

Resolving the Sky - Radio Interferometry: Past, Present and Future -RTS2012

April 17-20, 2012

Manchester, UK

\footnotetext{
* Speaker.

${ }^{\dagger}$ Green, Madsen, Banyer, Thakkar, Campbell-Wilson are affiliated with the ARC Centre of Excellence for All-sky Astrophysics (CAASTRO)
} 


\section{Introduction}

The Square Kilometre Array Molonglo Prototype (SKAMP) is a project using innovative technologies to transform the scientific capabilities of the University of Sydney's Molonglo Radio Telescope, located outside Canberra. The SKAMP project seeks to develop a new signal pathway on the existing mechanical infrastructure of the Molonglo Observatory Synthesis Telescope (MOST) to exploit wideband high-speed digital electronics. The project is presently completing its second stage, which will commission a new spectral line capability and produce more sensitive images of the sky than has been possible with MOST.

The telescope has an impressive history of producing panoramic sky surveys [4], [10], [11]. SKAMP represents a transformational upgrade to the telescope. The project has been approved as both a science and technology pathfinder instrument for the Square Kilometre Array (SKA), the next generation powerful radio telescope being planned by an international consortium. The value in utilising the Molonglo Telescope is that the costly infrastructure is already built, with known and tested performance capability, and that it has a large collecting area (approximately $1 \%$ of the SKA) and a record of expertise in low-cost high-volume production engineering.

\section{System Overview}

The telescope is an Earth rotation aperture synthesis instrument consisting of two cylindrical paraboloids, each $778 \times 12 \mathrm{~m}$, aligned EW to give a total extent of $1.6 \mathrm{~km}$. Each arm is subdivided into 176 separate elements with two independent signals being captured from each element. SKAMP-2 retains the front end configuration from MOST, so there are roughly 90 individual ring antennas detecting Right Hand Circular polarisation (IEEE definition) feeding each element. The signals are combined in phase, amplified by broadband uncooled low noise amplifiers and then transferred by coaxial cable to new digital receivers in shielded boxes under the telescope mesh reflectors. The receivers use 8-bit digitisation for better management of radio frequency interference (RFI) and to maximise image dynamic range. An optic fibre network provides timing signals and control lines and transfers the signals to the central control area for processing.

All intra- and inter-arm correlations are performed with a digital correlator and polyphase filterbank system, based on programmable logic chips (FPGAs). Signals are transferred via gigabit Ethernet switches to the control computer and data acquisition computers for analysis. The total data rate for all channels is $1.3 \mathrm{Gbps}$. A TB array will hold all visibilities from a complete image synthesis. Data are integrated and recorded every 32 seconds producing 6.7 TB per 12-hour observation. However, for SKAMP-2 the front end bandwidth is $1 / 3$ of what is planned for the full system with the new dual-polarisation feeds. This allows reduction of the size of the raw data cube to $2.2 \mathrm{~TB}$, for a field of view of $\sim 5 \mathrm{deg}^{2}$ and 4000 frequency channels.

Observations are conducted remotely and the regular geometry of the array means that while there are more than 67,000 baselines recorded, there are only 131 independent baseline pairs. This allows for a factor of about 30 in data compression following automated post observation calibration, to produce a data cube of $70 \mathrm{~GB}$ for each full synthesis in the SKAMP-2 configuration. There will be sufficient storage arrays to allow offline automated calibration and RFI mitigation algorithms to be applied simultaneously while the subsequent scheduled observation is being made. 


\begin{tabular}{|l|l|}
\hline Parameter & Performance Value \\
\hline Frequency range & $830-860 \mathrm{MHz}$ (fully processed) \\
Number of interferometer elements & 176 (with 2 outputs per element) \\
Collecting area & $18,000 \mathrm{~m}^{2}$ \\
Aperture efficiency & $50 \%$ \\
Number of baselines & $67,528(131$ independent $)$ \\
Number spectral channels & 4000 \\
System temperature & $\sim 65 \mathrm{~K}$ \\
Polarisation & $\mathrm{RHC}$ \\
Field of view & $\sim 5 \mathrm{deg}^{2}$ \\
Longest baseline & $1.6 \mathrm{~km}^{\circ}$ \\
Sky coverage & $<-30^{\circ}$ (full synthesis) \\
Spatial resolution & $43^{\prime \prime \times} 43^{\prime \prime} \operatorname{cosec} \delta$ ) \\
Spectral resolution & $2.5 \mathrm{~km} \mathrm{~s}^{-1}$ \\
$1 \sigma$ continuum sensitivity (12-hr synthesis) & $200 \mu \mathrm{Jy} \mathrm{beam}^{-1}$ \\
$1 \sigma$ spectral sensitivity (12-hr synthesis) & $6 \mathrm{mJy} \mathrm{beam}^{-1}$ \\
\hline
\end{tabular}

Table 1: Performance specifications for SKAMP-2

The process of baseline or frequency averaging, as needed by the science projects, is computationally simple and determined by the disk array speed. It is expected that all these processes will fit comfortably within the time of a 12-hour synthesis observation. It is planned that contiguous observing occurs for several days each week, interspersed with routine maintenance. An archival data array will be maintained on site and disk packs regularly exchanged with Sydney for backup storage.

The SKAMP project was planned in three stages. The first stage (SKAMP-1) is complete; during this stage the digital signal pathway was designed and the fundamental architecture of the data stream changed from recording fan beam voltages to recording spatial visibilities. Table 1 gives details of the performance specifications expected from the second stage of the project, SKAMP-2. Some of the parameters are set by the telescope infrastructure and are unchanged from operations as the MOST. During the final phase (SKAMP-3) the goal is to install new wide-band receivers with full polarisation capabilities.

\section{SKAMP-2 Progress}

For SKAMP-2 the only hardware component not yet completed is the FPGA-board for the digital receiver. Four prototype receivers have been field-tested since September 2011. Issues with instabilities and thermal performance have been resolved. The production run of these boards is expected to begin in July 2012. All other hardware components have been manufactured and installed, namely the optic fibre network, the timing signals and the filter bank and correlator boards. A diagram showing the status of the separate components is shown in Figure 1. 


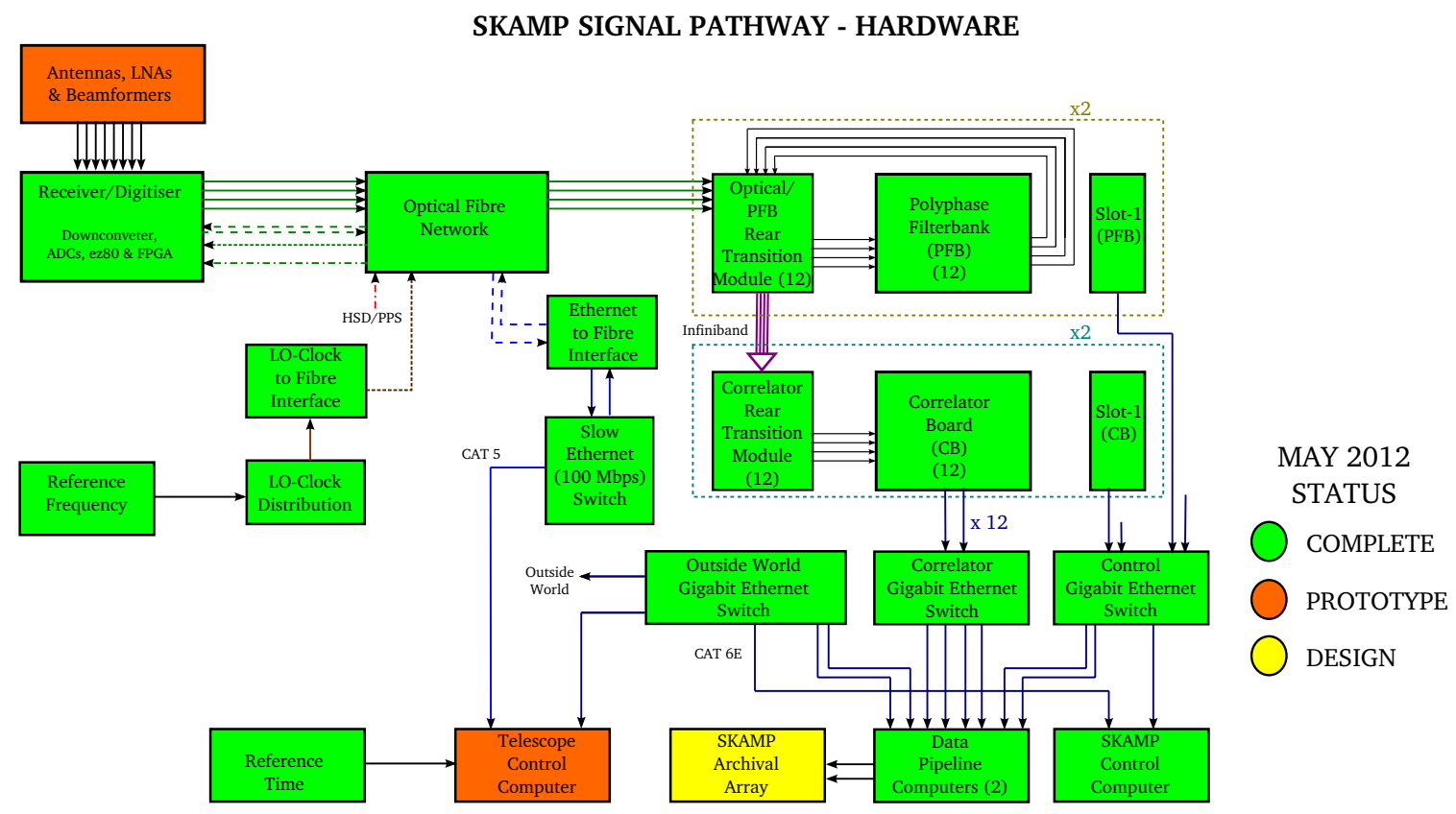

Figure 1: Status of completion for SKAMP-2 hardware components. The system of antennas, frontline amplifiers and beamformers is part of the future SKAMP-3 project



Figure 2: Screenshot of the real time diagnosis and visualisation tools, which can be used to interactively monitor the integrity of the data. 
Firmware to drive the principal digital systems is in development and undergoing testing. Software programs for monitoring and control of telescope operations, the observer interface and data acquisition are in various stages of development. Integration and commissioning of the complete signal pathway is planned for completion by September 2013. Valuable data visualisation tools have been developed for this project, but are expected to have wider application.

The SKAMP-2 system has the capacity to correlate signals from 67,528 baselines over the full bandwidth of 12,480 frequency channels, every 32 seconds. In order to diagnose and monitor the system efficiently, we have developed tools to visualise interactively the correlator output in near real time. An animated colour map is created that shows the amplitude and phase of every unique antenna pair as a function of integration time or frequency channel. Tools that display and analyse the change in amplitude and phase with time or frequency channel for a particular antenna pair are also available. These tools enable any error in the phase, delays or receiver gains to be quickly identified and corrected. Figure 2 is a screenshot of these tools displaying data from the antenna elements of the SKAMP-1 system, showing the status of all the baseline pairs and a time display of signal amplitude and phase.

\section{Survey Science}

\subsection{Transients and Variables}

The time variable radio sky reveals a number of phenomena that are not well understood. There is growing interest in detecting radio emission from supernovae, orphan gamma-ray bursts, extreme scattering events, and other exotica. The large field of view and sensitivity of SKAMP is well suited to the discovery of transient events and in particular, to the sustained followup of individual sources. An example is SN1987A in the Large Magellanic Cloud, for which the MOST has the best sampled light curve to date [1] [12]. More recently, an archival study for variables and transient sources has been made using the MOST database by Bannister et al. [2], [3].

Figure 3 compares SKAMP-2 with other radio transient and variability studies (adapted from [5]). The two-epoch equivalent snapshot rate is shown as a function of flux density; upper limits are indicated by L-shaped patterns. The slanted grey lines indicate the anticipated rates and brightnesses for different kinds of transient systems. The snapshot rate for SKAMP-2 was evaluated under the assumption that one $5 \mathrm{deg}^{2}$ image is created every day for two years. The figure shows that SKAMP-2 will explore a large parameter space in the search for radio transient and variable sources.

\subsection{Gas assembly in galaxies}

Another key goal is to understand when and where the gas in the early Universe is converted to stars, by measuring the neutral hydrogen gas in absorption against distant bright radio continuum sources. At present there are few accurate measurements of the gas density over much of cosmic time [7]. With SKAMP-2 we have a small slice in redshift space $(0.66<\mathrm{z}<0.72)$ in which to conduct a blind survey. It is predicted there is a $0.6 \%$ chance that a given source will have HI absorption in our redshift band and typically there will be 7 sources per field of view with a flux density greater than $150 \mathrm{mJy}$. This is the intensity required to detect an absorption line of optical 




Figure 3: Capability of SKAMP-2 to detect variable sources; adapted from Frail et. al (2012).

depth 0.05 . Hence, to generate a sample of 50 sources, the blind survey would need to cover 1400 fields [8]. Results will be tested against the predictions of the different semi-analytical models for galaxy assembly.

\subsection{The ionised interstellar medium}

With the spectral line capability there is the possibility of observing several radio recombination lines in our frequency band. These thermal lines trace the ionised interstellar medium in the Galaxy. They are probes of physical processes and the energetics of the gas and can provide information about temperature, density and kinematics. At $843 \mathrm{MHz}$ the lines are best for measuring low density gas, with $n_{e}<100 \mathrm{~cm}^{-3}$ and Emission Measures around $700 \mathrm{~cm}^{-6} \mathrm{pc}$. A survey along the Galactic Plane to determine the distribution and kinematics of ionised hydrogen will be synergistic with the searches for variables [9].

\section{Discussion}

The SKAMP-2 project is nearing practical completion and commissioning of the total signal pathway is expected from the end of 2012. There are plans to increase the training capacity of the telescope with opportunities for engineering students to learn first-hand about the technical challenges facing a large radio interferometer. There is a related technology research program to be- 
come an internationally recognised platform for developing automated techniques and algorithms to counter the pervasive and invasive problem of radio frequency interference in radio astronomy. Understanding the impact of transient man-made signals and how they might fundamentally differ from astrophysical signals will be necessary for all new radio telescopes [6]. Although the Molonglo Telescope is a mature instrument it has the capability to contribute in both the scientific and technological domains as we move closer to realising the SKA project.

\section{References}

[1] Ball, L., Crawford, D. F., Hunstead, R. W., Klamer, I. \& McIntyre, V. J., Radio Supernova 1987A at $843 \mathrm{MHz}$, Astrophysical Journal, 549, 599, 2001

[2] Bannister, K. W., Murphy, T., Gaensler, B. M., Hunstead, R. W., \& Chatterjee, S., A 22-yr southern sky survey for transient and variable radio sources using the Molonglo Observatory Synthesis Telescope, Monthly Notices of the Royal Astronomical Society, 412, 634, 2011a

[3] Bannister, K. W., Murphy, T., Gaensler, B. M., Hunstead, R. W. \& Chatterjee, S., Erratum: A 22-yr southern sky survey for transient and variable radio sources using the Molonglo Observatory Synthesis Telescope, Monthly Notices of the Royal Astronomical Society, 418, 2813, 2011b

[4] Bock, D. C.-J., Large, M. I. \& Sadler, E. M., SUMSS: A Wide-Field Radio Imaging Survey of the Southern Sky. I. Science Goals, Survey Design, and Instrumentation, Astronomical Journal, 117, 1578,1999

[5] Frail, D. A., Kulkarni, S. R., Ofek, E. O., Bower, G. C. \& Nakar, E., A Revised View of the Transient Radio Sky, Astrophysical Journal, 747, 70, 2012

[6] Fridman, P. A. \& Baan, W. A., RFI mitigation methods in radio astronomy, Astronomy \& Astrophysics, 378, 327, 2001

[7] Johnston, S. et al., Science with the Australian Square Kilometre Array Pathfinder, Publications Astronomical Society of Australia, 24, 174, 2007

[8] Green, A. J., HI absorption studies of galaxies and the SKA Molonglo Prototype (SKAMP) Project, POS (MRU2007) 011, 2007a

[9] Green, A. J., Murphy, T., Campbell-Wilson, D., Kesteven, M. J. \& Bunton, J., New Galactic ISM results and progress towards low-frequency spectroscopy, Highlights of Astronomy, 14, 368, 2007b

[10] Mauch, T., Murphy, T., Buttery, H. J., Curran, J., Hunstead, R.W., Piestrzynski, B., Robertson, J. G., \& Sadler, E. M., SUMSS: a wide-field radio imaging survey of the southern sky - II. The source catalogue, Monthly Notices of the Royal Astronomical Society 342, 1117, 2003

[11] Murphy, T., Mauch, T., Green, A.J., Hunstead, R.W., Piestrzynska, B., Kels, A.P. \& Sztajer, P., The second epoch Molonglo Galactic Plane survey: compact source catalogue, Monthly Notices of the Royal Astronomical Society, 382, 382, 2007

[12] Turtle, A.J., Campbell-Wilson, D., Bunton, J.D., Jauncey, D.L., Kesteven, M.J., Manchester, R. N., Norris, R. P., Storey, M. C. \& Reynolds, J. E., A prompt radio burst from supernova 1987A in the Large Magellanic Cloud, Nature, 327, 38, 1987 